\title{
Anticoagulants' Safety and Effectiveness in General Practice: A Nationwide Prospective Cohort Study
}

Paul Frappé, $M D, P b D$

Joël Cogneau, MD

Yoann Gaboreau, MD

Nathan Abenbaïm, MD

Marc Bayen, MD

Claude Guichard

Jean-Pierre Jacquet, MD

François Lacoin, MD

Sandra Liébart, MD

Laurent Bertoletti, MD, $\mathrm{PbD}$

Jean-Luc Bosson, MD, PbD

for the CACAO study investigators*

*Investigators participating in the CACAO study are listed in Supplemental Appendix, available at http://www.AnnFamMed.org/ content/18/2/131/suppl/DC1/.

Conflicts of interest: authors report none.

\section{CORRESPONDING AUTHOR}

Paul Frappé, MD, PhD

Department of General Practice, Faculty

of Medicine Jacques Lisfranc

Campus Santé Innovations

10 rue de la Marandière

42270 Saint Priest en Jarez, France

paul.frappe@univ-st-etienne.fr

\begin{abstract}
PURPOSE Most real-world studies on anticoagulants have been based on health insurance databases or performed in secondary care. The aim of this study was to compare safety and effectiveness between patients treated with vitamin $\mathrm{K}$ antagonists (VKAs) and patients treated with direct oral anticoagulants (DOACs) in a general practice setting.
\end{abstract}

METHODS The CACAO study (Comparison of Accidents and their Circumstances with Oral Anticoagulants) is a multicenter prospective cohort study conducted among ambulatory patients taking an oral anticoagulant. Participants were patients from the study's cross-sectional phase receiving oral anticoagulants because of nonvalvular atrial fibrillation, for secondary prevention of venous thromboembolism, or both. They were followed as usual for 1 year by their general practitioners, who collected data on changes in therapy, thromboembolic events, bleeding, and deaths. All events were adjudicated by an independent committee. We used a propensity score and a Cox regression model to derive hazard ratios.

RESULTS Between April and December 2014, a total of 3,082 patients were included. At 1 year, 42 patients (1.7\%) had experienced an arterial or venous event; 151 (6.1\%) had experienced bleeding, including 47 (1.9\%) who experienced major bleeding; and 105 (4.1\%) had died. There was no significant difference between the VKA and DOAC groups regarding arterial or venous events, or major bleeding. The VKA group had a lower risk of overall bleeding (hazard ratio $=0.65 ; 95 \% \mathrm{Cl}$, $0.43-0.98$ ) but twice the risk of death (hazard ratio $=1.98 ; 95 \% \mathrm{Cl}, 1.15-3.42$ ).

CONCLUSIONS VKAs and DOACs had fairly similar safety and effectiveness in general practice. The substantially higher incidence of deaths with VKAs is consistent with known data from health insurance databases and calls for further research to understand its cause.

Ann Fam Med 2020;18:131-138. https://doi.org/10.1370/afm.2495.

\section{INTRODUCTION}

$\mathrm{O}$ ral anticoagulants are widely prescribed in general practice, where their main indications are atrial fibrillation and venous thromboembolic disease. These medications have well-proven effectiveness for those indications but are associated with increased bleeding risk. ${ }^{1}$ In ambulatory care, anticoagulants are involved in $12 \%$ of suspected adverse drug reactions and are the leading cause of emergency department admission for such reactions, because of bleeding. ${ }^{2,3}$

The pattern of anticoagulant prescribing is changing. Direct oral anticoagulants (DOACs) have been developed as an alternative to vitamin K antagonists (VKAs). Since their introduction in 2009, DOACs have accounted for a steadily increasing share of the total, ${ }_{1}^{4}$ reaching $38.0 \%$ among all patients receiving anticoagulants in France as of 2016. ${ }^{1}$ The many available medications have diverse pharmacokinetic and pharmacodynamic profiles, leading to different treatment choices, taking into account potential medication interactions and patients' adherence, age, and renal function. ${ }^{1}$

Several real-world studies have provided results confirming those of phase 3 trials of DOACs, showing at least similar efficacy as VKAs 
and possibly better safety. ${ }^{1,5-14}$ Most of these studies, however, were based on health insurance databases, had a retrospective design, and were performed in secondary or tertiary care populations. Primary care clinicians continue to face practical issues in managing anticoagulant therapy, such as nonmajor bleeding, changes in anticoagulant therapy, patients' individual history, and adherence.

The aim of this study was to compare safety and effectiveness between VKAs and DOACs in a general practice setting.

\section{METHODS}

The CACAO study (Comparison of Accidents and their Circumstances with Oral Anticoagulants) is a multicenter prospective observational cohort study conducted by general practitioners throughout France among unselected ambulatory patients receiving an oral anticoagulant. The 463 investigators from 391 practices cover 290 rural or urban towns, and are distributed over 47 counties of France.

\section{Study Eligibility}

We identified participants in phase 1 of the CACAO study for follow-up. ${ }^{15}$ In that first, cross-sectional phase, every patient aged 18 years or older taking an oral anticoagulant and consulting a general practitioner investigator, for any reason, was eligible. Patients receiving injectable anticoagulants and those younger than the age of 18 years were excluded. Each investigator enrolled all consecutive eligible patients for 3 months, beginning between April and October 2014. To be included in the phase 2 follow-up study, patients from phase 1 had to have a nonvalvular atrial fibrillation or thromboembolic indication for anticoagulation, and to need anticoagulation for more than 3 months. Among patients receiving an anticoagulant for more than 1 year, every patient receiving a DOAC was matched to 3 patients receiving VKAs on sex, age, indication for anticoagulation, duration of anticoagulant therapy, and renal function.

\section{Data Collection}

During 1 year of follow-up, patients received usual care from their general practitioner, without any mandatory appointments. Every 3 months, general practitioners collected data on bleeding events according to the International Society on Thrombosis and Haemostasis classification ${ }^{16,17}$; on the thrombotic of ischemic stroke, deep vein thrombosis (DVT), pulmonary embolism (PE), and acute coronary syndrome; on death; and on changes to anticoagulant therapy and the reasons for changes.
General practitioners also collected items for the $\mathrm{CHA}_{2} \mathrm{DS}_{2}$-VASc score (congestive heart failure, hypertension, age, diabetes, previous stroke/transient ischemic attack-vascular ${ }^{18}$ and for the HAS-BLED score (hypertension, abnormal liver/renal function, stroke history, bleeding history or predisposition, labile international normalized ratio, elderly, drug/alcohol use). ${ }^{19}$ Both scores range from 0 to 9 , with higher scores indicating greater risk for stroke $\left(\mathrm{CHA}_{2} \mathrm{DS}_{2}-\mathrm{VASc}\right)$ or major bleeding (HAS-BLED). These scores were automatically calculated.

Data were collected anonymously on an electronic case report form. All events were adjudicated by an independent committee.

\section{Statistical Analysis}

Characteristics of the study population are reported as numbers and percentages, with means and standard deviations. Comparisons were performed using the $\chi^{2}$ or Fisher exact test for categorical variables and the Student $t$ test for continuous variables.

We calculated person-years of follow-up from the date of inclusion to the occurrence of first endpoint, death, or end of follow-up. Incidence rates were calcu-

\section{Figure 1. Flow diagram for the CACAO study.}

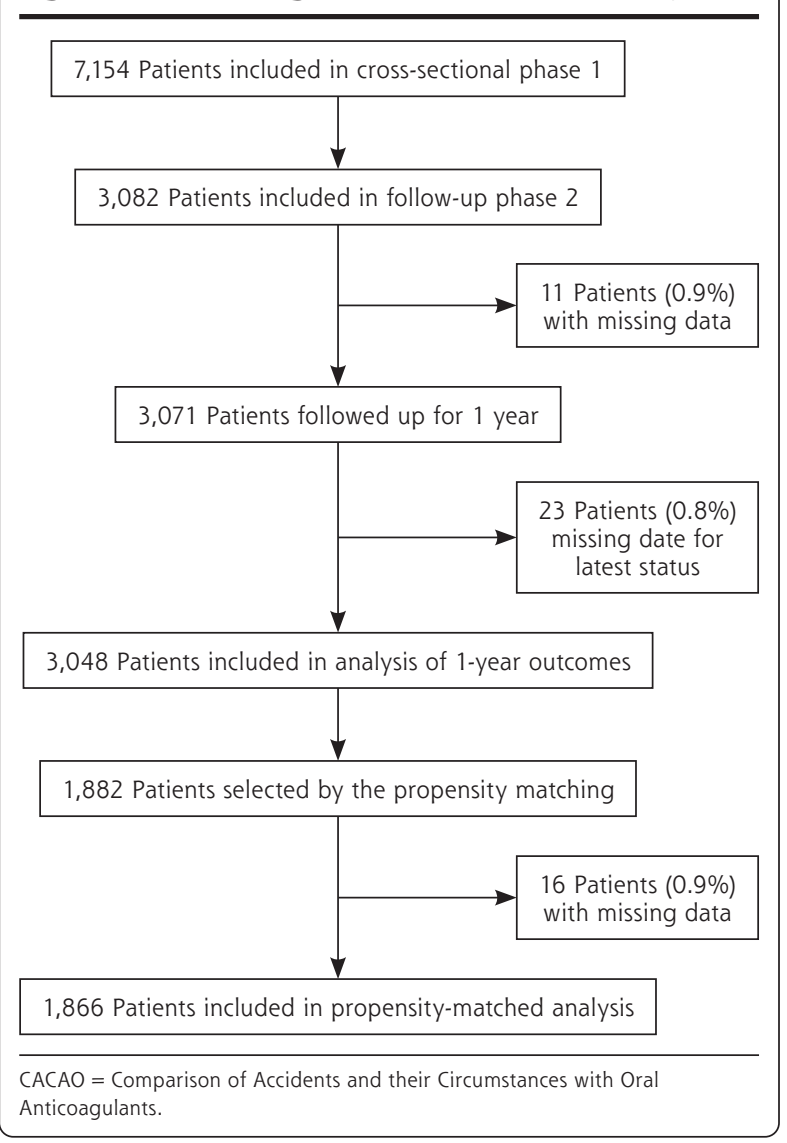


lated as number of events divided by person-years. No imputation of missing data was planned.

Occurrence of death, arterial or venous events, and bleeding during the year of follow-up was analyzed with the Kaplan-Meier method. Incidence rates and hazard ratios (HRs) by anticoagulant class (VKA vs DOAC) were calculated with a Cox regression model. For arterial or venous events, and for hemorrhagic events, we used the Fine and Gray method ${ }^{20}$ to calculate absolute risk of events taking into account the competing risk of death.

To further compare VKA- and DOAC-treated patients, we performed a second matching using a propensity score. The propensity model included age (4 age groups) and sex; personal history of hypertension, DVT and/or PE, diabetes mellitus, symptomatic heart failure, peripheral arterial disease, bleeding requiring hospitalization, or cancer $r_{i}$ Timed Up and Go test as estimated by the general practitioner ${ }_{i} \mathrm{CHA}_{2} \mathrm{DS}_{2}$-VASc score at the time of atrial fibrillation (0-1 vs $\geq 2)_{i}$ HAS-BLED score at the time of atrial fibrillation ( $\leq 3$ vs $>3$ ); renal failure (present vs absent); indication for anticoagulation (2 classes); associated antiplatelet or nonsteroidal anti-inflammatory drug therapy $y_{i}$ number of associated medications; duration of anticoagulant treatment ( $\leq 1$ year vs $>1$ year) , patient adherence as perceived by the general practitioner ( 2 classes); and general practitioner sex and age (continuous variable). Patients for whom matching was not possible were excluded from the propensity analysis.

We performed analyses using Stata version 15.0 (StataCorp, LLC). A 2 -sided $P<.05$ was considered significant.

\section{Ethics}

The CACAO study was approved by the ethical committee of the University Hospital of Saint-Etienne (IRBN112014/ CHUSTE). Its protocol is registered with ClinicalTrials.gov (NCT02376777). All patients received written information about the study, emphasizing their right to decline participation or to withdraw at any time. No written informed consent was required for inclusion.

\section{RESULTS}

Between April and December 2014, the 463 general practitioners included 7,154 patients in the first phase of the study, of whom 3,082 were included in the follow-up phase: 1,946 patients $(63.1 \%)$ were receiving a VKA and 1,136 patients $(36.9 \%)$ were receiving a DOAC (Figure 1). Characteristics at the start of the follow-up phase were fairly well balanced between groups (Table 1).

Table 1. Characteristics of Patients at Start of Follow-Up $(N=3,082)$

\begin{tabular}{|c|c|c|c|c|}
\hline Characteristic & $\begin{array}{c}\text { VKA }^{\mathrm{a}} \\
(\mathrm{n}=1,946)\end{array}$ & $\begin{array}{c}\text { DOACa } \\
(n=1,136)\end{array}$ & $P$ & $\begin{array}{c}\text { Totala } \\
(\mathrm{N}=3,082)\end{array}$ \\
\hline \multicolumn{5}{|l|}{ Age } \\
\hline Mean (SD), y & $75.0(11.5)$ & $73.3(12.3)$ & $<.001$ & $74.4(11.8)$ \\
\hline$\geq 75$ years, No. (\%) & $1,138(58.5)$ & $599(52.7)$ & .002 & $1,737(56.4)$ \\
\hline Male, No. (\%) & $1,095(56.3)$ & $594(52.3)$ & .03 & $1,689(54.8)$ \\
\hline Weight, mean (SD), kg & $80.1(18.0)$ & $78.9(17.7)$ & .08 & 79.7 (17.9) \\
\hline \multicolumn{5}{|l|}{ Body mass index } \\
\hline Mean (SD), kg/m² & $28.8(5.8)$ & $28.4(5.6)$ & .13 & $28.6(5.7)$ \\
\hline$>30$ kg/m², No. (\%) & $576(35.7)$ & 336 (34.9) & .68 & $912(35.4)$ \\
\hline \multicolumn{5}{|l|}{ Medical history, No. (\%) } \\
\hline Hypertension & $1,367(70.3)$ & 757 (66.6) & .04 & $2,124(68.9)$ \\
\hline DVT and/or PE & $467(24.0)$ & $186(16.4)$ & $<.001$ & $653(21.2)$ \\
\hline Diabetes mellitus & $469(24.1)$ & $253(22.3)$ & .25 & $722(23.4)$ \\
\hline $\begin{array}{l}\text { Coronary heart disease and/ } \\
\text { or } \mathrm{Ml}\end{array}$ & $393(20.2)$ & $156(13.7)$ & $<.001$ & $549(17.8)$ \\
\hline Symptomatic heart failure & $336(17.3)$ & $141(12.4)$ & $<.001$ & $477(15.5)$ \\
\hline Stroke and/or TIA & $303(15.6)$ & $172(15.1)$ & .75 & $475(15.4)$ \\
\hline Peripheral arterial disease & $220(11.3)$ & $101(8.9)$ & .03 & $321(10.4)$ \\
\hline $\begin{array}{l}\text { Bleeding requiring } \\
\text { hospitalization }\end{array}$ & $146(7.5)$ & $54(4.8)$ & .003 & $200(6.5)$ \\
\hline Cancer & $65(3.3)$ & $27(2.4)$ & .13 & $92(3.0)$ \\
\hline $\begin{array}{l}\text { Timed Up and Go test as esti- } \\
\text { mated by GP, No. }(\%)^{b}\end{array}$ & & & $<.001$ & \\
\hline$<14$ seconds & $1,022(52.5)$ & $695(61.2)$ & & $1,717(55.7)$ \\
\hline $14-30$ seconds & $473(24.3)$ & $238(21.0)$ & & $711(23.1)$ \\
\hline$>30$ seconds & $229(11.8)$ & $108(9.5)$ & & $337(10.9)$ \\
\hline $\mathrm{CHA}_{2} \mathrm{DS}_{2}$-VASc score, No. $(\%)^{\mathrm{c}, \mathrm{d}}$ & & & .007 & \\
\hline 0 & $20(1.2)$ & $29(2.9)$ & & $49(1.9)$ \\
\hline 1 & $98(6.1)$ & $65(6.5)$ & & $163(6.2)$ \\
\hline$\geq 2$ & $1,499(92.7)$ & $900(90.5)$ & & $\begin{array}{r}2,399(91.9) \\
\text { continues }\end{array}$ \\
\hline
\end{tabular}

$2 \mathrm{~N}=2$ times the upper limit of normal; $3 \mathrm{~N}=3$ times the upper limit of normal; $\mathrm{ALT}=$ alanine aminotransferase; $\mathrm{AST}=$ aspartate aminotransferase; $\mathrm{CHA}_{2} \mathrm{DS}_{2}-\mathrm{VASC}=$ congestive heart failure, hypertension, age (>75 = 2 points), diabetes, previous stroke/transient ischemic attack (2 points)-vascular; DOAC = direct oral anticoagulants; DVT = deep vein thrombosis; GP = general practitioner; HAS-BLED = hypertension, abnormal liver/renal function, stroke history, bleeding history or predisposition, labile international normalized ratio, elderly, drug/alcohol use; $\mathrm{MI}=$ myocardial infarction; NSAID = nonsteroidal anti-inflammatory drug; $\mathrm{PE}=$ pulmonary embolism; TIA = transient ischemic attack; VKA = vitamin $\mathrm{K}$ antagonist.

a Denominators may be less than shown in column heads because of missing data for some characteristics. ${ }^{b}$ Longer time indicates poorer mobility.

c Calculated for 2,611 patients with nonvalvular atrial fibrillation.

d Scores range from 0 to 9; higher scores indicate greater stroke risk. 
Overall, 11 patients $(0.4 \%)$ were excluded from analysis because of missing data. Patients in the DOAC group as compared with counterparts in the VKA group more often had a definitive stopping of their anticoagulation $\left(12.0 \%\right.$ vs $\left.6.9 \%{ }_{i} P<.001\right)$ and at least 1 switch of anticoagulant class $(4.0 \%$ vs $2.2 \%$; $P=.005$ ) (Table 2). Among those switching, DOAC recipients more commonly did so because of intolerance $(17.8 \%$ vs $0 \% ; P=.006)$ or altered renal function $(26.7 \%$ vs $2.3 \% ; P=.001)$.

During the year of follow-up,

Table 1. Characteristics of Patients at Start of Follow-Up ( $\mathrm{N}=3,082)$ (continued)

\begin{tabular}{|c|c|c|c|c|}
\hline Characteristic & $\begin{array}{c}\text { VKA }^{a} \\
(n=1,946)\end{array}$ & $\begin{array}{c}\text { DOACa } \\
(n=1,136)\end{array}$ & $P$ & $\begin{array}{l}\text { Total }^{\mathrm{a}} \\
(\mathrm{N}=3,082)\end{array}$ \\
\hline HAS-BLED score, No. $(\%)^{\text {ce e }}$ & & & $<.001$ & \\
\hline$\leq 3$ & $1,293(80.0)$ & $883(88.8)$ & & $2,176(83.3)$ \\
\hline$>3$ & $324(20.0)$ & $111(11.2)$ & & $435(16.7)$ \\
\hline Renal failure (clearance), No. (\%) & & & .07 & \\
\hline None ( $\geq 60 \mathrm{~mL} / \mathrm{min})$ & $1,317(67.7)$ & $805(70.9)$ & & $2,122(68.9)$ \\
\hline Moderate $(30-60 \mathrm{~mL} / \mathrm{min})$ & $513(26.4)$ & $257(22.6)$ & & $770(25.0)$ \\
\hline Severe (15-30 mL/min) & $19(1.0)$ & $7(0.6)$ & & $26(0.8)$ \\
\hline Terminal (<15 mL/min) & $1(0.1)$ & $0(0)$ & & $1(0)$ \\
\hline Hepatic function, No. (\%) & & & .04 & \\
\hline $\begin{array}{l}\text { AST and/or ALT }>3 \mathrm{~N} \text { and/or } \\
\text { bilirubin }>2 \mathrm{~N}\end{array}$ & $12(0.6)$ & $2(0.2)$ & & $14(0.5)$ \\
\hline $\begin{array}{l}\text { AST and/or ALT }<3 \mathrm{~N} \text { and bili- } \\
\text { rubin }<2 \mathrm{~N}\end{array}$ & $1,434(73.7)$ & $875(77.0)$ & & $2,309(74.9)$ \\
\hline $\begin{array}{l}\text { Indication for anticoagulation, } \\
\text { No. (\%) }\end{array}$ & & & .001 & \\
\hline Nonvalvular atrial fibrillation & $1,619(83.2)$ & $994(87.5)$ & & $2,613(84.8)$ \\
\hline Treatment of DVT/PE & $327(16.8)$ & $142(12.5)$ & & $469(15.2)$ \\
\hline Anticoagulant, No. (\%) & & & $\cdots$ & \\
\hline Acenocoumarol & $113(5.8)$ & $\ldots$ & & $113(3.7)$ \\
\hline Apixaban & $\ldots$ & $66(5.8)$ & & $66(2.1)$ \\
\hline Dabigatran & $\ldots$ & $440(38.7)$ & & $440(14.3)$ \\
\hline Fluindione & $1,397(71.8)$ & $\ldots$ & & $1,397(45.3)$ \\
\hline Rivaroxaban & $\ldots$ & $630(55.5)$ & & $630(20.4)$ \\
\hline Warfarin & $436(22.4)$ & $\ldots$ & & $436(14.1)$ \\
\hline $\begin{array}{l}\text { Number of concomitant medica- } \\
\text { tions with risk for interaction, } \\
\text { No. }(\%)^{\dagger}\end{array}$ & & & .82 & \\
\hline None & $798(41.0)$ & $478(42.1)$ & & $1,276(41.4)$ \\
\hline 1 & $795(40.9)$ & $452(39.8)$ & & $1,247(40.5)$ \\
\hline$\geq 2$ & $353(18.1)$ & $206(18.1)$ & & $559(18.1)$ \\
\hline $\begin{array}{l}\text { Duration of anticoagulant } \\
\text { treatment }>1 \text { year, No. (\%) }\end{array}$ & $1,446(74.3)$ & $582(51.3)$ & $<.001$ & $2,028(65.8)$ \\
\hline $\begin{array}{l}\text { Patient adherence as perceived } \\
\text { by GP }\end{array}$ & & & .10 & \\
\hline Not or not very adherent & $103(5.4)$ & $76(6.8)$ & & $179(5.9)$ \\
\hline Rather or completely adherent & $1,820(94.6)$ & $1,041(93.2)$ & & $2,861(94.1)$ \\
\hline
\end{tabular}

$2 \mathrm{~N}=2$ times the upper limit of normal; $3 \mathrm{~N}=3$ times the upper limit of normal; $\mathrm{ALT}=$ alanine aminotransferase; $\mathrm{AST}=$ aspartate aminotransferase; $\mathrm{CHA}_{2} \mathrm{DS}_{2}-\mathrm{VASC}=$ congestive heart failure, hypertension, age ( $>75=2$ points), diabetes, previous stroke/transient ischemic attack (2 points)-vascular; DOAC = direct oral anticoagulants; DVT = deep vein thrombosis; GP = general practitioner; HAS-BLED = hypertension, abnormal liver/renal function, stroke history, bleeding history or predisposition, labile international normalized ratio, elderly, drug/alcohol use; $\mathrm{MI}=$ myocardial infarction; NSAID = nonsteroidal anti-inflammatory drug; $\mathrm{PE}=$ pulmonary embolism; TIA = transient ischemic attack; VKA = vitamin $\mathrm{K}$ antagonist.

a Denominators may be less than shown in column heads because of missing data for some characteristics.

${ }^{b}$ Longer time indicates poorer mobility.

' Calculated for 2,611 patients with nonvalvular atrial fibrillation.

d Scores range from 0 to 9; higher scores indicate greater stroke risk.

e Scores range from 0 to 9; higher scores indicate greater risk for major bleeding.

${ }^{f}$ Concomitant medications from medical records were statins, amiodarone, antiplatelet agents, serotonin reuptake inhibitors, fibrate, verapamil, NSAIDs, quinidine, carbamazepine, tacrolimus, cyclosporine, anticoagulants, systemic antifungal agents, rifampicin, protease inhibitors.
105 patients $(4.1 \%)$ died $_{i} 151$ (6.1\%) experienced bleeding events, including 47 (1.9\%) who experienced major bleeding events; and $42(1.7 \%)$ experienced arterial or venous events: 14 ischemic strokes, 11 myocardial infarctions, 9 DVTs, 5 PEs, 3 transient ischemic attacks, and 1 arterial thrombosis of the lower limb. Relative to the DOAC group, the VKA group had a lower risk of any bleeding ( $\mathrm{HR}=0.67 ; 95 \% \mathrm{CI}, 0.49-0.92)$ driven mainly by a lower risk of minor bleeding $(\mathrm{HR}=0.49 ; 95 \%$ CI, 0.25-0.96), but a higher risk of death $(\mathrm{HR}=2.37 ; 95 \% \mathrm{CI}$, 1.45-3.85) (Table 3).

The propensity-matched analysis was based on 1,866 patients: 935 (50.1\%) were receiving a VKA and 931 (49.9\%) were receiving a DOAC (Table 4). The receiver operating characteristic curve model for the propensity model had an area under the curve of 0.675 , indicating moderately good matching. Sixteen patients $(0.9 \%)$ were excluded from analysis because of missing data. Relative to the DOAC group, the VKA group had a lower risk of any bleeding $(\mathrm{HR}=0.65 ; 95 \% \mathrm{CI}, 0.43$ $0.98)$ but a higher risk of death $(\mathrm{HR}=1.98 ; 95 \% \mathrm{CI}, 1.15-3.42)$ (Table 5).

\section{DISCUSSION}

\section{Main Findings}

We report the results of the first French national prospective cohort study of ambulatory patients receiving oral anticoagulant therapy, a study designed and conducted by and for general 
Table 2. Changes in Anticoagulation During Year of Follow-Up, by Anticoagulant Class at Start of Follow-Up $(\mathrm{N}=3,071)$

\begin{tabular}{|c|c|c|c|c|}
\hline $\begin{array}{l}\text { Anticoagulation } \\
\text { Change }\end{array}$ & $\begin{array}{c}\text { VKA, } \\
\text { No. }(\%) \\
(n=1,941)\end{array}$ & $\begin{array}{c}\text { DOAC, } \\
\text { No. }(\%) \\
(n=1,130)\end{array}$ & $P$ Value & $\begin{array}{c}\text { Total, } \\
\text { No. }(\%) \\
(\mathrm{N}=3,071)\end{array}$ \\
\hline Definitive stop & $134(6.9)$ & $135(12.0)$ & $<.001$ & $269(8.8)$ \\
\hline$\geq 1$ temporary stop & $126(6.5)$ & $67(5.9)$ & .54 & $193(6.3)$ \\
\hline$\geq 1$ switch of drug class & $43(2.2)$ & $45(4.0)$ & .005 & $88(2.9)$ \\
\hline \multicolumn{5}{|l|}{ Reason for switch ${ }^{a}$} \\
\hline Unstable INR & $26(60.5)$ & $0(0)$ & $\ldots$ & $26(29.6)$ \\
\hline Patient demand & $11(25.6)$ & $9(20.0)$ & .53 & $20(22.7)$ \\
\hline Change of indication & $0(0)$ & $2(4.4)$ & .50 & $2(2.3)$ \\
\hline Medication intolerance & $0(0)$ & $8(17.8)$ & .006 & $8(9.1)$ \\
\hline Adherence problem & $4(9.3)$ & $1(2.2)$ & .20 & $5(5.7)$ \\
\hline Renal function & $1(2.3)$ & $12(26.7)$ & .001 & $13(14.8)$ \\
\hline Hepatic function & $0(0)$ & $2(4.4)$ & .50 & $2(2.3)$ \\
\hline Thrombosis event & $0(0)$ & $3(6.7)$ & .24 & $3(3.4)$ \\
\hline Bleeding event & $2(4.7)$ & $8(17.8)$ & .09 & $10(11.4)$ \\
\hline Other & $8(18.6)$ & $9(20.0)$ & .87 & $17(19.3)$ \\
\hline
\end{tabular}

any bleeding $(6.1 \%$ in our study vs $2.85 \%$ to $4.83 \%$ in previous studies), major bleeding $(1.9 \%$ vs $1.12 \%$ to $4.04 \%)$, and mortality ( $4.1 \%$ vs $4.62 \%$ to $7.41 \%) .{ }^{11,13,21}$ All phase III trials and real-world cohort studies have shown a higher rate of death in VKA groups, but their hazard ratios were not always significant and were mostly smaller than ours, ${ }^{1,11,13,21-24}$ which was 1.98 (95\% CI, 1.15-3.42). This elevated risk was mainly due to sudden deaths and deaths from malignancies, infections, and undetermined causes, and not to bleeding, suggesting it may stem from an external cause independent of anticoagulation therapy, such as our recruitment in primary care; other contextual factors missed in our matching process; or a lack of statistical power in our study.

Our VKA group had a lower

practitioners. During the year of follow-up, about $2 \%$ of patients had an arterial or venous event; roughly $6 \%$ had bleeding, including $2 \%$ with major bleeding; and slightly more than $4 \%$ died. After propensity matching, there was no significant difference between patients receiving VKAs and patients receiving DOACs regarding arterial or venous events and major bleeding. The VKA group had a $35 \%$ lower risk of any bleeding but almost double the risk of all-cause mortality; the latter difference was not due to deaths from bleeding.

\section{Comparison With Existing Literature}

Our participants' age and sex were similar to those of patients in previous cohort studies (mean age $=74$ years in our cohort vs 70 years in previous cohorts; female $=45.2 \%$ vs $39 \%$ to $47 \%$ ), but the rate of renal impairment was higher in our study ( $25.8 \%$ vs $4.5 \%$ to $10.1 \%) .{ }^{11,13,21}$ Risk scores vary widely in the previous literature: the proportion of patients with a $\mathrm{CHA}_{2} \mathrm{DS}_{2}$-VASc score of 2 or greater ranges from $59 \%$ to $90 \%$, whereas it was $91.9 \%$ in our study, and the proportion with a HASBLED score of 3 or greater varies from $6.3 \%$ to $41 \%$, whereas the proportion with a score exceeding 3 was $16.7 \%$ in our study.

Incidence rates were similar to those of cohorts studies for
Table 3. One-Year Outcomes, by Anticoagulant Class at Outcome ( $N=3,048 ; 2,641$ Person-Years)

\begin{tabular}{|c|c|c|c|c|}
\hline Outcome & $\begin{array}{c}\text { VKA, } \\
\text { No. }(\%) \\
(n=1,932)\end{array}$ & $\begin{array}{c}\text { DOAC, } \\
\text { No. }(\%) \\
(n=1,116)\end{array}$ & $\begin{array}{c}\text { Total, } \\
\text { No. }(\%) \\
(\mathrm{N}=3,048)\end{array}$ & HR $(95 \% \mathrm{Cl})$ \\
\hline $\begin{array}{l}\geq 1 \text { arterial or venous } \\
\text { event }\end{array}$ & $26(1.6)$ & $16(1.8)$ & $42(1.7)$ & $0.89(0.48-1.66)$ \\
\hline Any bleeding & $83(5.2)$ & $68(7.6)$ & 151 (6.1) & $0.67(0.49-0.92)$ \\
\hline Major bleeding & 27 (1.7) & $20(2.2)$ & 47 (1.9) & $0.75(0.42-1.33)$ \\
\hline $\begin{array}{l}\text { Nonmajor but clinically } \\
\text { relevant bleeding }\end{array}$ & $40(2.5)$ & $30(3.3)$ & $70(2.8)$ & $0.74(0.46-1.18)$ \\
\hline Minor bleeding & $16(1.0)$ & $18(2.0)$ & $34(1.3)$ & $0.49(0.25-0.96)$ \\
\hline All-cause death & $85(5.2)$ & $20(2.2)$ & $105(4.1)$ & $2.37(1.45-3.85)$ \\
\hline
\end{tabular}


form. In France, there are no anticoagulation clinics, and anticoagulation monitoring and international normalized ratio management are usually performed by general practitioners, limiting the impact of the absence of mandatory follow-up appointments. Data were collected from patients' medical records, and there was no audit to verify that general practitioners did not miss any events. In France, however, patients have to register with a unique general practitioner or receive financial penalties, which limits that potential measurement bias. It is possible that physicians who agreed to participate in the study were more engaged in continuing medical education, medical research, and/or the anticoagulant topic than the average general practitioner. This possible recruitment bias should have a tendency to lead to underestimation of our outcomes, which are nevertheless important for practice. All events were adjudicated by a central committee, which reinforces the credibility of our results.

Given the context of continuing medical education of investigators, which imposed a minimum and a maximum cluster size, a complete initial matching was not possible. Inclusion of an average of just 4.8 patients per practice, however, obviated the need to adjust for clustering. The observed statistical power was low, especially regarding bleeding events and arteriovenous events. Propensity matching reduced the sample size but showed a good quality of the model (area under the curve $=0.675$ ), which further reinforces our findings.

\section{Conclusions}

In this study, arteriovenous events and major bleeding events did not differ between patients receiving VKAs and patients receiving DOACs. The near doubling of mortality risk in the VKA group as compared with the DOAC group is consistent with known data from health insurance databases and calls for further research to explore its origin.

\section{Table 4. Characteristics of Patients in the Propensity-Matched Sample $(\mathrm{N}=1,882)$}

\begin{tabular}{|c|c|c|c|c|}
\hline Characteristic & $\begin{array}{c}\text { VKA } \\
(n=941)\end{array}$ & $\begin{array}{c}\text { DOAC } \\
(n=941)\end{array}$ & $\begin{array}{c}P \\
\text { Value }\end{array}$ & $\begin{array}{c}\text { Total } \\
(\mathrm{N}=1,882)\end{array}$ \\
\hline Age group, No. (\%) & & & .61 & \\
\hline$<50$ years & $45(4.8)$ & $40(4.3)$ & & $85(4.5)$ \\
\hline $50-70$ years & $232(24.7)$ & $254(27.0)$ & & $486(25.8)$ \\
\hline $70-80$ years & $301(32.0)$ & $303(32.2)$ & & $604(32.1)$ \\
\hline$\geq 80$ years & $363(38.6)$ & $344(36.7)$ & & 707 (37.6) \\
\hline Male, No. (\%) & $503(53.5)$ & $504(53.6)$ & .96 & $1,007(53.5)$ \\
\hline \multicolumn{5}{|l|}{ Medical history, No. (\%) } \\
\hline Hypertension & $644(68.4)$ & $640(68.0)$ & .84 & $1,284(68.2)$ \\
\hline DVT and/or PE & $162(17.2)$ & $169(18.0)$ & .67 & $331(17.6)$ \\
\hline Diabetes mellitus & $220(23.4)$ & $214(22.8)$ & .74 & $434(23.1)$ \\
\hline \multicolumn{5}{|l|}{ Coronary heart disease and/or MI } \\
\hline Symptomatic heart failure & $131(13.9)$ & $133(14.1)$ & .89 & $264(14.0)$ \\
\hline \multicolumn{5}{|l|}{ Stroke and/or TIA } \\
\hline Peripheral arterial disease & $304(32.3)$ & $312(33.1)$ & .69 & $616(32.7)$ \\
\hline Bleeding requiring hospitalization & $46(4.9)$ & $49(5.2)$ & .75 & $95(5.1)$ \\
\hline Cancer & $21(2.2)$ & $25(2.7)$ & .55 & $46(2.4)$ \\
\hline $\begin{array}{l}\text { Timed Up and Go test as estimated by } \\
\text { GP, No. (\%) }\end{array}$ & & & .83 & \\
\hline$<14$ seconds & $546(58.0)$ & $556(59.1)$ & & $1,102(58.6)$ \\
\hline $14-30$ seconds & $213(22.6)$ & $206(21.9)$ & & $419(22.3)$ \\
\hline$>30$ seconds & $90(9.6)$ & $96(10.2)$ & & $186(9.9)$ \\
\hline $\mathrm{CHA}_{2} \mathrm{DS}_{2}$-VASc score, No. (\%) & & & .74 & \\
\hline $0-1$ & $77(8.2)$ & $81(8.6)$ & & $158(8.4)$ \\
\hline$\geq 2$ & $864(91.8)$ & $860(91.4)$ & & $1,724(91.6)$ \\
\hline HAS-BLED score, No. (\%) & & & .89 & \\
\hline$\leq 3$ & $827(87.9)$ & $829(88.1)$ & & 1,656 (88.0) \\
\hline$>3$ & $114(12.1)$ & $112(11.9)$ & & $226(12.0)$ \\
\hline Renal failure, No. (\%) & & & .49 & \\
\hline No (clearance $\geq 60 \mathrm{~mL} / \mathrm{min}$ ) & $629(66.8)$ & $652(69.3)$ & & $1,281(68.1)$ \\
\hline Yes (clearance <60 mL/min) & $255(27.1)$ & $233(24.8)$ & & $488(25.9)$ \\
\hline Indication for anticoagulation, No. (\%) & & & .89 & \\
\hline Nonvalvular atrial fibrillation & $811(86.2)$ & $809(86.0)$ & & $1,620(86.1)$ \\
\hline Treatment of DVT/PE & $130(13.8)$ & $132(14.0)$ & & $262(13.9)$ \\
\hline \multicolumn{5}{|l|}{$\begin{array}{l}\text { Concomitant medications with risk for } \\
\text { interaction, No. (\%) }\end{array}$} \\
\hline$\geq 2$ medications & $165(17.5)$ & $164(17.4)$ & .98 & $329(17.5)$ \\
\hline NSAID & $13(1.4)$ & $12(1.3)$ & .84 & $25(1.3)$ \\
\hline Antiplatelet agent & $80(8.5)$ & $75(8.0)$ & .68 & $155(8.2)$ \\
\hline $\begin{array}{l}\text { Duration of anticoagulant therapy }>1 \\
\text { year, No. (\%) }\end{array}$ & $552(58.7)$ & $572(60.8)$ & .35 & $1,124(59.7)$ \\
\hline Age of GP, mean (SD), y & $49.8(10.0)$ & $50.2(9.7)$ & .36 & $50.0(9.9)$ \\
\hline Sex of GP, male, No. (\%) & $694(73.8)$ & $695(73.9)$ & .96 & $1,389(73.8)$ \\
\hline $\begin{array}{l}\text { Patient adherence as perceived by GP, } \\
\text { No. (\%) }\end{array}$ & & & .93 & \\
\hline Not or not very adherent & $63(6.7)$ & $64(6.8)$ & & $127(6.8)$ \\
\hline Rather or completely adherent & $878(93.3)$ & $877(93.2)$ & & $1,755(93.6)$ \\
\hline
\end{tabular}


Table 5. One-Year Outcomes in the Propensity-Matched Sample, by Anticoagulant Class at Outcome ( $\mathrm{N}=1,866 ; 1,618$ Person-Years)

\begin{tabular}{|c|c|c|c|c|}
\hline Outcome & $\begin{array}{c}\text { VKA, } \\
\text { No. }(\%)\end{array}$ & $\begin{array}{l}\text { DOAC, } \\
\text { No. }(\%)\end{array}$ & $\begin{array}{l}\text { Total, } \\
\text { No. }(\%)\end{array}$ & $\operatorname{HR}(95 \% \mathrm{CI})$ \\
\hline$\geq 1$ arterial or venous event & $15(1.9)$ & $14(1.9)$ & $29(1.9)$ & $1.00(0.48-2.07)$ \\
\hline Any bleeding & $38(4.9)$ & $55(7.4)$ & $93(6.1)$ & $0.65(0.43-0.98)$ \\
\hline Major bleeding & $8(1.0)$ & $17(2.3)$ & $25(1.6)$ & $0.45(0.19-1.03)$ \\
\hline $\begin{array}{l}\text { Nonmajor but clinically relevant } \\
\text { bleeding }\end{array}$ & $21(2.7)$ & $22(2.9)$ & $43(2.8)$ & $0.90(0.50-1.64)$ \\
\hline Minor bleeding & $9(1.1)$ & $16(2.1)$ & $25(1.6)$ & $0.52(0.23-1.18)$ \\
\hline All-cause death & $40(5.0)$ & $19(2.5)$ & $59(3.8)$ & $1.98(1.15-3.42)$ \\
\hline Sudden death/dysrhythmia & $10(1.3)$ & $1(0.1)$ & $\cdots$ & $\ldots$ \\
\hline Heart failure & $4(0.5)$ & $3(0.4)$ & $\cdots$ & $\cdots$ \\
\hline Myocardial infarction & $1(0.1)$ & $0(0)$ & $\cdots$ & $\cdots$ \\
\hline $\begin{array}{l}\text { Ischemic stroke/systemic embolism } \\
\text { (other than embolic stroke) }\end{array}$ & $0(0)$ & $2(0.3)$ & $\cdots$ & $\cdots$ \\
\hline Hemorrhagic stroke & $1(0.1)$ & $1(0.1)$ & $\ldots$ & $\cdots$ \\
\hline Extracranial hemorrhage & $1(0.1)$ & $5(0.7)$ & $\cdots$ & $\cdots$ \\
\hline Other vascular death & $1(0.1)$ & $1(0.1)$ & $\cdots$ & $\cdots$ \\
\hline Malignancy & $7(0.9)$ & $1(0.1)$ & $\cdots$ & $\cdots$ \\
\hline Infection & $8(1.0)$ & $0(0)$ & $\ldots$ & $\cdots$ \\
\hline Respiratory & $0(0)$ & $2(0.3)$ & $\ldots$ & $\cdots$ \\
\hline Trauma/accidental & $0(0)$ & $1(0.1)$ & $\cdots$ & $\cdots$ \\
\hline Hepatobiliary/liver failure & $0(0)$ & $1(0.1)$ & $\ldots$ & $\ldots$ \\
\hline All other nonvascular death & $0(0)$ & $0(0)$ & $\cdots$ & $\cdots$ \\
\hline Undetermined death & $7(0.9)$ & $1(0.1)$ & $\ldots$ & $\ldots$ \\
\hline
\end{tabular}

Acknowledgments: The authors are indebted to Claire Eychenne, Carole Rolland, and Céline Vermorel for their assistance in data management and analysis. We thank the members of the Primary Care Unit of the University of Geneva (Switzerland) for their editorial advice.

Clinical Trial Registration: ClinicalTrials. gov NCT02376777

Supplemental materials: Available at http://www.AnnFamMed.org/ content/18/2/131/suppl/DC1/.

\section{References}

1. Haute autorité de santé. Commission de la transparence. Rapport d'évaluation des médicaments anticoagulants oraux [Evaluation report of oral anticoagulant drugs]. https://www.has-sante.fr/ portail/upload/docs/application/ pdf/2018-02/rapport_reev_aco_cte val234_2018-02-09_15-38-37_999.pdf. Published 2018. Accessed Feb 11, 2020.

2. Kane-Gill SL, Van Den Bos J, Handler SM. Adverse drug reactions in hospital and ambulatory care settings identified using a large administrative database. Ann Pharmacother. 2010;44(6): 983-993.
To read or post commentaries in response to this article, see it online at http://www.AnnFamMed.org/content/18/2/131.

Key words: anticoagulants; general practice; cohort studies; patient safety; medical records; primary care; practice-based research

Submitted December 10, 2018; submitted, revised, June 6, 2019; accepted July 29, 2019.

Author affiliations: Department of General Practice, University of Saint-Etienne, Saint-Etienne, France (Frappé); Inserm UMR 1059, Sainbiose DVH, University of Saint-Etienne, Saint-Etienne, France (Frappé, Bertoletti); Inserm CIC-EC 1408, Saint-Etienne, France (Frappé, Bertoletti); Primary Care Unit, University of Geneva, Geneva, Switzerland (Frappé); Institut de Recherche en Médecine Générale, Paris, France (Cogneau, Abenhaïm, Guichard, Lacoin); Department of General Practice, Grenoble Alpes University, Grenoble, France (Gaboreau, Jacquet); TIMC-IMAG UMR 5525, Grenoble Alpes University, Grenoble, France (Gaboreau, Bosson); Department of General Practice, University of Lille, Lille, France (Bayen); Ambulatory Care Consultation Unit, CHU de Saint-Etienne, Saint-Etienne, France (Liébart); Department of Vascular Medicine and Therapeutics, CHU de Saint-Etienne, Saint-Etienne, France (Bertoletti).

Funding support: The CACAO study was supported by the French government (continuing professional development of investigators).

Disclaimer: The views expressed are solely those of the authors and do not necessarily represent official views of the authors' affiliated institutions or funders.

Previous presentation: This work been presented at the Wonca World Conference; October 17-21, 2019; Seoul, Korea.
3. Budnitz DS, Lovegrove MC, Shehab N, Richards CL. Emergency hospitalizations for adverse drug events in older Americans. N Engl J Med. 2011;365(21):2002-2012.

4. Ten Cate V, Ten Cate H, Verheugt FWA. The Global Anticoagulant Registry in the FIELD-Atrial Fibrillation (GARFIELD-AF): Exploring the changes in anticoagulant practice in patients with non-valvular atrial fibrillation in the Netherlands. Neth Heart J. 2016;24(10):574-580.

5. Graham DJ, Reichman ME, Wernecke M, et al. Cardiovascular, bleeding, and mortality risks in elderly Medicare patients treated with dabigatran or warfarin for nonvalvular atrial fibrillation. Circulation. 2015;131(2):157-164.

6. Kodani $\mathrm{E}$, Atarashi $\mathrm{H}$, Inoue $\mathrm{H}$, Okumura $\mathrm{K}$, Yamashita T, Origasa $\mathrm{H}$; J-RHYTHM Registry Investigators. Beneficial effect of non-vitamin $\mathrm{k}$ antagonist oral anticoagulants in patients with nonvalvular atrial fibrillation - results of the J-RHYTHM Registry 2. Circ J. 2016;80(4): 843-851.

7. Villines TC, Schnee J, Fraeman K, et al. A comparison of the safety and effectiveness of dabigatran and warfarin in non-valvular atrial fibrillation patients in a large healthcare system. Thromb Haemost. 2015;114(6):1290-1298.

8. Seeger JD, Bykov K, Bartels DB, Huybrechts K, Zint K, Schneeweiss $S$. Safety and effectiveness of dabigatran and warfarin in routine care of patients with atrial fibrillation. Thromb Haemost. 2015;114(6): 1277-1289.

9. López-López JA, Sterne JAC, Thom HHZ, et al. Oral anticoagulants for prevention of stroke in atrial fibrillation: systematic review, network meta-analysis, and cost effectiveness analysis. BMJ. 2017;359: j5058.

10. Jun M, Lix LM, Durand M, et al; Canadian Network for Observational Drug Effect Studies (CNODES) Investigators. Comparative safety of direct oral anticoagulants and warfarin in venous thromboembolism: multicentre, population based, observational study. BMJ. 2017;359:j4323. 
11. Larsen TB, Skjøth F, Nielsen PB, Kjældgaard JN, Lip GY. Comparative effectiveness and safety of non-vitamin $\mathrm{K}$ antagonist oral anticoagulants and warfarin in patients with atrial fibrillation: propensity weighted nationwide cohort study. BMJ. 2016;353:i3189.

12. Maura G, Blotière PO, Bouillon K, et al. Comparison of the shortterm risk of bleeding and arterial thromboembolic events in nonvalvular atrial fibrillation patients newly treated with dabigatran or rivaroxaban versus vitamin $\mathrm{K}$ antagonists: a French nationwide propensity-matched cohort study. Circulation. 2015;132(13): 1252-1260.

13. Huisman MV, Rothman KJ, Paquette M, et al; GLORIA-AF Investigators. Two-year follow-up of patients treated with dabigatran for stroke prevention in atrial fibrillation: Global Registry on LongTerm Antithrombotic Treatment in Patients with Atrial Fibrillation (GLORIA-AF) registry. Am Heart J. 2018;198:55-63.

14. Vinogradova Y, Coupland C, Hill T, Hippisley-Cox J. Risks and benefits of direct oral anticoagulants versus warfarin in a real world setting: cohort study in primary care. BMJ. 2018;362:k2505.

15. Frappé $P$, Cogneau J, Gaboreau $Y$, et al. Areas of improvement in anticoagulant safety. Data from the CACAO study, a cohort in general practice. PLoS One. 2017;12(4):e0175167.

16. Kaatz S, Ahmad D, Spyropoulos AC, Schulman S; Subcommittee on Control of Anticoagulation. Definition of clinically relevant nonmajor bleeding in studies of anticoagulants in atrial fibrillation and venous thromboembolic disease in non-surgical patients: communication from the SSC of the ISTH. J Thromb Haemost. 2015;13(11): 2119-2126.
17. Schulman S, Kearon C; Subcommittee on Control of Anticoagulation of the Scientific and Standardization Committee of the International Society on Thrombosis and Haemostasis. Definition of major bleeding in clinical investigations of antihemostatic medicinal products in non-surgical patients. J Thromb Haemost. 2005;3(4):692-694.

18. Lip GY, Nieuwlaat R, Pisters R, Lane DA, Crijns HJ. Refining clinical risk stratification for predicting stroke and thromboembolism in atrial fibrillation using a novel risk factor-based approach: the Euro Heart Survey on atrial fibrillation. Chest. 2010;137(2):263-272.

19. Pisters R, Lane DA, Nieuwlaat R, de Vos CB, Crijns HJ, Lip GY. A novel user-friendly score (HAS-BLED) to assess 1-year risk of major bleeding in patients with atrial fibrillation: the Euro Heart Survey. Chest. 2010;138(5):1093-1100.

20. Fine JP, Gray RJ. A proportional hazards model for the subdistribution of a competing risk. J Am Stat Assoc. 1999;94(446):496-509.

21. Yao X, Abraham NS, Sangaralingham LR, et al. Effectiveness and safety of dabigatran, rivaroxaban, and apixaban versus warfarin in nonvalvular atrial fibrillation. J Am Heart Assoc. 2016;5(6):e003725.

22. Connolly SJ, Ezekowitz MD, Yusuf S, et al; RE-LY Steering Committee and Investigators. Dabigatran versus warfarin in patients with atrial fibrillation. N Engl J Med. 2009;361(12):1139-1151.

23. Patel MR, Mahaffey KW, Garg J, et al; ROCKET AF Investigators. Rivaroxaban versus warfarin in nonvalvular atrial fibrillation. $N$ Engl J Med. 2011;365(10):883-891.

24. Granger CB, Alexander JH, McMurray JJV, et al; ARISTOTLE Committees and Investigators. Apixaban versus warfarin in patients with atrial fibrillation. N Engl J Med. 2011;365(11):981-992. 